\title{
Les bras et le ventre. Une sociologie politique des plantations industrielles dans le Cameroun contemporain
}

Thèse de doctorat (PhD), université Paris 1 Panthéon-Sorbonne, 2019, $953 \mathrm{p}$.

\section{Guillaume Vadot}

\section{OpenEdition Journals}

Édition électronique

URL : https://journals.openedition.org/anthropodev/1107

DOI : 10.4000/anthropodev. 1107

ISSN : 2553-1719

Éditeur

Presses universitaires de Louvain

\section{Édition imprimée}

Date de publication : 1 décembre 2020

Pagination : 205-206

ISBN : 978-2-39061-078-6

ISSN : 2276-2019

Référence électronique

Guillaume Vadot, «Les bras et le ventre. Une sociologie politique des plantations industrielles dans le Cameroun contemporain », Anthropologie \& développement [En ligne], 51 | 2020, mis en ligne le 01 décembre 2020, consulté le 12 février 2022. URL : http://journals.openedition.org/anthropodev/1107 ; DOI : https://doi.org/10.4000/anthropodev.1107

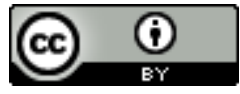

La revue Anthropologie \& développement est mise à disposition selon les termes de la Licence Creative Commons Attribution 4.0 International. 


\title{
Les bras et le ventre. Une sociologie politique des plantations industrielles dans le Cameroun contemporain
}

\author{
Guillaume Vadot ${ }^{1}$ \\ Thèse de doctorat $(P h D)$, université Paris 1 Panthéon-Sorbonne, \\ 2019, $953 \mathrm{p}$.
}

Les plantations industrielles forment le premier secteur d'emploi privé au Cameroun, et couvrent d'immenses surfaces. Malgré leur importance pour la production du territoire, il s'agit d'espaces peu étudiés. Ailleurs, ce sont les enjeux fonciers bien plus que ceux liés au travail qui ont concentré l'attention des études sur le capitalisme agricole dans les Sud. Quelles sont les trajectoires biographiques et professionnelles de celles et ceux qui y travaillent, dans leur diversité ? Comment s'y crée-t-il du quotidien et des espaces sociaux localisés? Comment les complexes de plantations parviennent-ils à tenir comme organisations bureaucratiques ? Quelle est leur contribution à la formation de l'État ?

La thèse répond à ces questions en s'appuyant sur trois monographies comparées, portant sur trois sites choisis parmi les quatre plus grands complexes agro-industriels du pays (deux entreprises privées, l'une sucrière et l'autre hévéacultrice, et une société publique pratiquant la polyculture). Chacune est alimentée par une enquête ethnographique qui a combiné l'observation directe du travail et des lieux de vie avec de nombreux entretiens (257), ainsi que par le dépouillement de vastes corpus documentaires et par un travail photographique.

Le manuscrit est organisé en deux parties. La première, « Vivre et travailler dans une plantation industrielle ", est consacrée à l'étude de la main-d'œuvre (tous statuts et toutes catégories confondus), en croisant sociologie des classes populaires, du travail et de l'emploi. La seconde, "La grande plantation comme terroir singulier du politique ", s'intéresse aux conflits et aux processus de bureaucratisation qui forment les racines locales de l'État.

La thèse appréhende les grandes plantations comme des espaces sociaux localisés, et établit que ces derniers sont marqués avant tout par la présence populaire. En reconstituant, à partir d'un large échantillon, les trajectoires de salarisation suivies par les ouvriers

\footnotetext{
${ }^{1}$ E-mail : guillaume.vadot@sciencespo.fr
} 
et ouvrières, elle rend compte de l'univers tactique habité par ces derniers et dernières, et de sa relative fragmentation selon le positionnement de l'expérience de la plantation dans les itinéraires biographiques. Elle s'attache à explorer les pratiques de travail dans leur quotidienneté, et décrit les logiques à travers lesquelles les corps sont mis en jeu par cette expérience. Les régimes de classement qui irriguent solidarités et différenciations, et notamment la fabrique locale du genre et de l'ethnicité, sont restitués dans un va-et-vient entre temps et lieux du travail et du dehors (quartier, associations, jardins). Cela permet d'éclairer comment se construisent les identifications ouvrières locales, minoritaires mais incontournables. L'exposé décrit également les tensions qui traversent le groupe des cadres et agents de maîtrise, en insistant aussi bien sur son ascendant local que ses fragilités, et s'intéresse aux significations de la division racialisée qui séparent cadres locaux et expatriés.

Ce premier ensemble de résultats sert alors à éclairer les processus sociaux qui permettent à l'entreprise de plantations de tenir et de s'incarner comme organisation, et qui contribuent localement à la formation de l'autorité. L'analyse se nourrit de l'observation de chaque complexe dans sa matérialité : procédures routinisées, circulation de papiers, entretien des frontières. Partout, les couches intermédiaires stabilisées jouent un rôle clé pour faire exister ces mécanismes. La spatialisation propre à chaque entreprise donne cependant lieu à d'importantes variations dans les modes locaux de gouvernementalité et donc corrélativement d'insubordination. Les incessants conflits fonciers et du travail nourrissent des mobilisations qui sont à l'origine de processus d'officialisation d'une série d'interactions au sein des plantations. La défense des "autochtones" (garantie par la constitution) autant que celle de l'« emploi » (item clé du discours politique) forment des causes qui légitiment des positions de représentants intégrées à la scène politique locale à travers les chefferies, les organes locaux du parti au pouvoir, les syndicats ou encore les mairies. La thèse contribue ainsi à la connaissance de la manière dont se forment, dans la confrontation entre ordre productif et ordre politique, des topographies politiques locales singulières mais intégrées au territoire camerounais.

La thèse défend aussi trois positionnements plus transversaux. Elle plaide pour que l'analyse du politique en Afrique tienne plus compte des processus sociaux liés au travail et à la production, en dialogue avec une boîte à outils incontournable mais qui a plutôt focalisé son attention sur les phénomènes d'appropriation et de consommation. Elle contribue aux débats qui animent la sociologie du travail en défendant l'importance de tenir compte des matérialités, de développer une sensibilité ethnographique intersectionnelle, mais aussi de s'intéresser aux mobilités. Enfin, elle ambitionne de reconstruire comme objet scientifique le travail salarié subalterne en Afrique, en allant au-delà des expectatives associées à la modernité comme des limites de la reconstruction opérée par la critique de cette dernière : l'ampleur des dynamiques de salarisation en cours sur le continent ouvre en effet de nouveaux chantiers. 